\title{
Triple versus monoterapia antibiótica en apendicitis complicada: ensayo clínico abierto, Instituto Hondureño de Seguridad Social, Tegucigalpa.
}

\author{
Triple versus antibiotic monotherapy for complicated appendicitis: \\ open clinical trial, Instituto Hondureño de Seguridad Social, Tegucigalpa.
}

\author{
Karen Santos, ${ }^{1}$ José Lizardo, ${ }^{2}$ Marco T. Luque. ${ }^{3}$ \\ 1Médico Residente Tercer Año Postgrado Pediatría, Facultad de Ciencias Médicas, Universidad Nacional Autónoma de Honduras, período 2011-2013. Actualmente \\ Médico Especialista, Centro Regional La Ceiba, Instituto Hondureño de Seguridad Social (IHSS); Hospital General Atlántida; La Ceiba. \\ ${ }^{2}$ Médico Especialista en Cirugía Pediátrica, Hospital de Especialidades IHSS, Tegucigalpa. \\ ${ }_{3}^{3}$ Médico Especialista en Infectología Pediátrica, Hospital de Especialidades IHSS; Hospital Escuela; Tegucigalpa.
}

\begin{abstract}
RESUMEN. Antecedentes: En Honduras no se dispone de evidencia publicada sobre diferencias en eficacia de esquemas terapéuticos basados en antibióticos para el manejo post-quirúrgico para apendicitis aguda complicada en niños. Objetivo: Determinar la eficacia y seguridad de triple versus monoterapia antibiótica en pacientes pediátricos con apendicitis aguda complicada, Hospital de Especialidades, Instituto Hondureño de Seguridad Social (IHSS), Tegucigalpa, 2011-2013. Métodos: Ensayo clínico abierto, dos esquemas terapéuticos: Triple Terapia (Ampicilina+Amikacina+Clindamicina, GrupoTT) y Ertapenem (GrupoE) durante 7 días. Se incluyeron pacientes con diagnóstico de apendicitis aguda complicada y sometidos a apendicectomía abierta. Los pacientes fueron evaluados en Consulta Externa, 7 días post-quirúrgicos. Se obtuvo aprobación por Comité de Etica en Investigación IHSS. Se utilizó prueba Chi-cuadrado, Riesgo Relativo, intervalo de confianza de $95 \%$ y valor de $p<0.05$ para determinar diferencias entre grupos. Resultados: Se incluyeron 58 pacientes en GrupoTT y 29 en Grupo E, edad media fue 7.3 años (IC95\% 6.7-7.9) GrupoTT y 8 años (IC95\% 7-9) GrupoE. La evolución promedio del cuadro clínico fue 31.6 horas GrupoTT y 43.8 horas GrupoE $(p=0.034)$. No se encontró diferencias significativas respecto a efectos adversos de importancia clínica, complicaciones postoperatorias, estancia intrahospitalaria en días, o necesidad de readmisión por recurrencia/complicación posterior al alta. Discusión: La monoterapia con ertapenem presentó similar eficacia y seguridad que la triple terapia con Ampicilina+Amikacina+Clindamicina empleada actualmente en el tratamiento del paciente pediátrico con apendicitis complicada. El estudio está limitado por que los dos grupos de estudio fueron diferentes en la evolución de la enfermedad.
\end{abstract}

Palabras clave: Antibacterianos, Apendicectomía, Apendicitis, Complicaciones posoperatorias.

\section{INTRODUCCIÓN}

La apendicitis aguda es la inflamación apendicular sintomática, clasificándose como perforada por cuadro clínico 0 identificación de fecalito o visualización de la perforación. ${ }^{1,2} \mathrm{Es}$ la causa más frecuente de cirugía abdominal de emergencia en niños, contabilizando en Estados Unidos de América (EUA) más de un tercio de todas las hospitalizaciones anuales por dolor abdominal en $<18$ años y $80 \%$ de las emergencias quirúrgicas pediátricas. ${ }^{1,2}$ El riesgo a lo largo de la vida para niños es $8.7 \%$ y $6.7 \%$ para niñas. La incidencia aumenta de $1-2$ por cada 10,000 niños $<4$ años hasta $25 / 10,000$ niños mayores, hay pequeña predominancia estacional en los meses de verano, aumentan el riesgo de apendicitis la dieta baja en fibra y antecedente familiar de apendicitis; se someterán a apendicectomía 4/1000 niños anualmente, distribuyéndose con menor frecuencia en $<5$ años y máxima entre 12-18 años. ${ }^{3,4}$

Recibido: 10-11-2018; Aceptado para publicación 11-5-2019

Dirección para correspondencia: Dra. Karen Santos

Correo electrónico: krensantos22@yahoo.com

Conflictos de interés. Los autores declaran no tener conflictos de interés en relación a este artículo.

DOI: https://doi.org/10.5377/rmh.v87i2.11904
La patogénesis inicia con obstrucción luminal apendicular entre la base cecal y la punta, por cuerpo extraño como apendicolito, inflamación o tejido linfoide intramural, induciendo edema, congestión y distensión mucosa con translocación de la flora colónica normal con infiltrado inflamatorio y progresión a celulitis de la pared colónica, que al abarcar la pared induce necrosis y gangrena. ${ }^{3}$ La sospecha clínica inicia el proceso de diagnóstico, reportando un estudio utilidad pronóstica del Score Pediátrico de apendicitis, valores de sensibilidad, especificidad y valores predictivos encima de $90 \% .{ }^{5} \mathrm{La}$ incidencia de perforación es de $20-40 \%$ y se identifica al momento de la cirugía, el pico de incidencia se presenta en lactantes con $70-95 \%$ de casos disminuyendo con la edad hasta $10-20 \%$ en la adolescencia; se observa mayor riesgo de perforación en los casos con cuadro mayor de 36 horas de evolución, pertenecer a minorías étnicas y bajo nivel socioeconómico. ${ }^{3,4}$

El diagnóstico diferencial en algunos casos puede ser un reto para el clínico, auxiliándose con algunas pruebas de laboratorio, radiografía, ultrasonido y tomografía. ${ }^{3,4,6}$ La mortalidad ha dejado de ser significativa, pasando la problemática actual a la prevención de morbilidad, en donde la perforación se asocia 
a peritonitis localizada, que puede gangrenarse y abscedarse, con riesgo de sepsis por anaerobios Gram positivos como $B$. fragilis, Clostridium, Peptoestreptococcus, así como Gram negativos como Escherichia coli, Pseudomonas, Enterobacter, Klebsiella sp., ${ }^{3,-11}$ El manejo inicial es médico y consiste en ampicilina, amikacina y clindamicina o metronidazol, y quirúrgico; hay evidencia en la literatura sobre la eficacia y seguridad de la administración de monoterapia con antibióticos de amplio espectro. ${ }^{3,9-13}$

Actualmente en Honduras no se dispone de evidencia publicada sobre las diferencias en eficacia de esquemas o regímenes terapéuticos basados en antibióticos ni balance alguno sobre el costo-beneficio de los mismos en la población pediátrica, lo que justifica la implementación de un estudio clínico que determine y compruebe lo establecido en la literatura mundial de acuerdo a nuestro medio hospitalario e idiosincrasia. El presente estudio se realizó con el objetivo de Determinar la eficacia y seguridad del Ertapenem versus triple terapia antibiótica en pacientes pediátricos con apendicitis complicada, Hospital de Especialidades, Instituto Hondureño de Seguridad Social (IHSS), en el período de Julio 2011 a Julio 2013.

\section{MÉTODOS}

Se realizó un ensayo clínico para comparar dos esquemas de tratamiento antibiótico, triple terapia estándar versus monoterapia alternativa, que incluyó pacientes pediátricos con edad entre 1 y 18 años con diagnóstico de apendicitis aguda complicada/perforada atendidos por especialista de la institución e ingresados en el Servicio de Cirugía Pediátrica del Hospital de Especialidades del IHSS entre Julio 2011 y Julio 2013. El diagnóstico de apendicitis se basó en criterios clínicos y laboratoriales reconocidos internacionalmente ${ }^{12}$. Se definió apendicitis complicada como el diagnóstico por especialista en cirugía pediátrica con o sin hallazgos descritos en el reporte de histopatología. Se excluyeron los casos con co-morbilidad importante identificada o conocida antes del ingreso o durante el mismo.

La muestra fue estimada mediante Epidat en base a una diferencia de $20 \%$ en la proporción de complicaciones en cada grupo, obteniendo una muestra mínima de 55 casos para cada grupo de tratamiento, con una significancia estadística de $5 \%$ y potencia de $80 \%$. Los pacientes se incluyeron mediante muestreo sistemático alternando los grupos de tratamiento. El Grupo de tratamiento estándar se trató con triple terapia I.V. de Ampicilina 100 mg/kg/día más Amikacina 15 mg/kg/día más Clindamicina 25-40 mg/kg/día. El grupo de monoterapia se trató con Ertapenem I.V. $30 \mathrm{mg} / \mathrm{kg} / \mathrm{día}$.

La información se captó por aplicación de encuesta mediante entrevista directa por el equipo investigador con el padre/ responsable de los pacientes, complementándose la información con datos de laboratorio y evolutivos en el expediente clínico. Todos los pacientes fueron evaluados en consulta externa 7 días después del alta hospitalaria. Se consideró la variable dependiente de eficacia la complicación postquirúrgica de etiología infecciosa relacionada con la patología o el tratamiento quirúrgico (e.g. infección de herida quirúrgica, absceso de pared, etc.) y de seguridad la identificación de efectos adversos de importancia clínica, que a su vez se definió como cualquier morbilidad asociada con el antibiótico(s) administrado. El cálculo de la proporción de casos nuevos se realizó dividiendo el número de diagnósticos de apendicitis diagnosticados entre el número total de ingresos en el período del estudio.

El procesamiento de la información se realizó introduciendo los datos recopilados en una base de datos diseñada y generada en Epi-Info 2000 (CDC, Atlanta, Georgia, USA, 2010), que se utilizó también para generar listados, cuadros y cruces divariados. Para comparación entre grupos se utilizó el programa StatCalc 1.0 (CDC, Atlanta, Georgia, USA, 1993) determinándose el valor de $p<0.05$ como prueba de independencia, con tablas de comparación de categorías (2x2), y usando la prueba Chi-cuadrado, se utilizó diferencia de medias para variables continuas y riesgo relativo para variables de desenlace.

El protocolo fue aprobado por el Comité de Etica en Investigación del Hospital de Especialidades del IHSS, Tegucigalpa. En todos los casos se obtuvo consentimiento informado escrito por el padre, madre, o responsable del paciente. La información personal de los participantes fue manejada de forma confidencial por parte del equipo de investigación.

\section{RESULTADOS}

Se incluyeron 58 pacientes en el grupo Triple Terapia y 29 pacientes en el grupo Ertapenem. La media de edad en Grupo Triple Terapia fue 7.3 años (IC95\% 6.7 - 7.9) y en Grupo Ertapenem fue 8 años (IC95\% 7.0 - 9.0) ( $p=0.07)$. No se encontró diferencia entre grupos respecto a edad, estrato de edad, sexo, ambiente de residencia (Cuadro 1). El tiempo de evolución del cuadro clínico al llegar al hospital fue 31.6 horas en el Grupo Triple Terapia y de 43.8 horas en el grupo Ertapenem ( $p=0.034)$.

La sintomatología más frecuentemente consignada fue similar en ambos grupos. En orden de frecuencia fue dolor localizado, náuseas/vómitos, fiebre, rebote en Fosa llíaca Derecha (FID), anorexia, dolor epigástrico y signo de Rovsing positivo (Cuadro 2). La mayor parte de casos fueron sometidos a cirugía de apéndice a las 24 horas del ingreso 95\% (55) en el Grupo Triple Terapia y $90 \%$ (26) en Grupo Ertapenem, sin diferencia estadística significativa (Cuadro 3). De acuerdo a los hallazgos intraoperatorios, la proporción de casos con perforación fue $100 \%$ de casos en ambos grupos (Cuadro 3). La proporción de complicaciones postquirúrgicas de importancia fue $7 \%$ (4) en Grupo Triple Terapia y de $21 \%$ (6) en Grupo Ertapenem, ocupando los primeros lugares en frecuencia en ambos grupos absceso de pared (4) necesidad de cambio de antibiótico (3), sepsis (2), peritonitis (2), no encontrándose diferencia entre grupos $(p>0.05)$ (Cuadro 3 ). Las complicaciones se asociaron 3 veces más a los pacientes que fueron tratados con monoterapia 
Cuadro 1. Características sociodemográficas de los pacientes pediátricos sometidos a triple terapia y monoterapia, ensayo clínico, IHSS, Tegucigalpa, 20112013.

\begin{tabular}{lccc}
\hline Características & $\begin{array}{c}\text { Triple Terapia } \\
\mathbf{n = 5 8} \\
\mathbf{N}(\%)\end{array}$ & $\begin{array}{c}\text { Monoterapia } \\
\mathbf{n = 2 9} \\
\mathbf{N}(\%)\end{array}$ & $\begin{array}{c}\text { Valor de } \\
\mathbf{p}\end{array}$ \\
\cline { 1 - 2 } Edad (años) & $0(0.0)$ & $1(3.0)$ & NS \\
$1-2$ & $11(19.0)$ & $3(10.0)$ & \\
$2-5$ & $46(79.0)$ & $24(83.0)$ & \\
$5-10$ & $0(0.0)$ & $1(3.0)$ & \\
$10-15$ & $1(2.0)$ & $0(0.0)$ & \\
$16-18$ & & & \\
Sexo & $33(57.0)$ & $22(76.0)$ & 0.084 \\
Masculino & $25(43.0)$ & $7(24.0)$ & \\
Femenino & & & NS \\
Ambiente residencia & $57(98.0)$ & $29(100.0)$ & \\
Urbano & $1(2.0)$ & $0(0.0)$ & \\
Rural & & & \\
\hline
\end{tabular}

NS= No significativo.
(RR 3.0, IC95\% 0.9-9.8), aunque esta diferencia no fue estadísticamente significativa $(p=0.07)$.

La media de estancia intrahospitalaria fue para el Grupo Triple Terapia 5.8 días (5.0 -6.6) y para el Grupo Ertapenem fue 7.1 días (6.0-8.2), sin diferencias entre grupos de comparación $(p=0.05)$, así como respecto a tasas de readmisión por complicaciones postquirúrgicas posteriores al alta y diagnosticadas en la Consulta Externa (3.5\% versus 3.5\%), de donde se realizó la referencia a la Sala de Hospitalización. No se presentó mortalidad en ninguno de los grupos de estudio.

\section{DISCUSIÓN}

Este es el primer estudio en Honduras en el área pediátrica en el que se compara la eficacia y seguridad del tratamiento estándar de triple terapia con la monoterapia antibiótica para la apendicitis aguda complicada. El hallazgo más importante de este estudio es que se no se encontró diferencia significativa en cuanto eficacia y seguridad de la monoterapia comparada con la triple terapia en pacientes pediátricos con apendicitis aguda complicada, ya que no se encontró diferencias estadísticamente significativas respecto al riesgo de complicaciones infecciosas postquirúrgicas en el grupo tratado durante 7 días con ampicilina más amikacina más clindamicina/metronidazol comparado con el manejo antibiótico con Ertapenem, similar a lo encontrado en otros estudios. ${ }^{3,7-11}$ Se ha discutido que ambos esquemas muestran igual eficacia antibiótica, pero también se ha argumentado sobre las ventajas que ofrece el proceso de manejo logístico y de administración de un solo fármaco comparado con el esfuerzo del personal, cadena logística, administración, y cumplimiento de la administración de tres fármacos, que indirectamente se asocia a disminución en costos y errores hospitalarios. ${ }^{5}$

La tasa de perforación reportada en apendicitis es entre $30-74 \% .13-15$ En nuestro estudio solo se incluyeron casos perforados (Cuadro 3). Las complicaciones se presentaron en $6.9 \%$ (4) en el Grupo Triple Terapia y en $20.7 \%$ (6) en el Grupo Ertapenem. Aunque estas diferencias no fueron estadísticamente significativas, demuestran una tendencia de mayor numero de complicaciones en el Grupo Ertapemen. Estas frecuencias identificadas son menores a lo reportado por otros autores con aproximadamente un tercio de casos que presentan absceso abdominal/infec- 
Cuadro 3. Características quirúrgicas y evolutivas de los pacientes pediátricos sometidos a triple terapia y monoterapia, ensayo clínico, IHSS, Tegucigalpa, 2011-2013.

\begin{tabular}{|c|c|c|c|}
\hline Características quirúrgicas/evolutivas & $\begin{array}{c}\text { Triple Terapia } \\
\mathrm{n}=58(\%)\end{array}$ & $\begin{array}{c}\text { Monoterapia } \\
\mathrm{n}=29(\%)\end{array}$ & $\begin{array}{l}\text { Valor } \\
\text { de } p\end{array}$ \\
\hline $\begin{array}{l}\text { Apendicectomía realizada en días } \\
\text { a partir de llegada a emergencia }\end{array}$ & & & NS \\
\hline 0 & $55(95.0)$ & $26(90.0)$ & \\
\hline 1 & $2(3.0)$ & $2(7.0)$ & \\
\hline 3 & $0(0.0)$ & $1(3.0)$ & \\
\hline 4 & $1(2.0)$ & $0(0.0)$ & \\
\hline \multicolumn{4}{|l|}{ Hallazgos intraoperatorios } \\
\hline Apéndice Perforado & $58(100.0)$ & $29(100)$ & \\
\hline Complicaciones postquirúrgicas & & & 0.057 \\
\hline $\mathrm{Si}$ & $4(7.0)$ & $6(21.0)$ & \\
\hline No & $54(93.0)$ & $23(79.0)$ & \\
\hline \multicolumn{4}{|l|}{ Tipo de complicación ( $n=15)$} \\
\hline Absceso de pared & $3(5.0)$ & $1(3.0)$ & \\
\hline Sepsis & $0(0.0)$ & $2(7.0)$ & \\
\hline Peritonitis & $1(2.0)$ & $1(3.0)$ & \\
\hline Infección del sitio quirúrgico & $0(0.0)$ & $1(3.0)$ & \\
\hline Absceso pélvico & $0(0.0)$ & $1(3.0)$ & \\
\hline Fiebre & $1(2.0)$ & $1(3.0)$ & \\
\hline Necesidad de cambio de antibiótico & $1(2.0)$ & $3(10.0)$ & \\
\hline Días de estancia intrahospitalaria & & & 0.066 \\
\hline$<5$ días & $20(34.0)$ & $2(7.0)$ & \\
\hline$>5$ días & $38(66.0)$ & $27(93.0)$ & \\
\hline \multicolumn{4}{|l|}{ Condición de egreso } \\
\hline Vivo & $58(100.0)$ & $29(100.0)$ & \\
\hline Readmisión & & & NS \\
\hline $\mathrm{Si}$ & $2(3.0)$ & $1(3.0)$ & \\
\hline No & $56(97.0)$ & $28(97.0)$ & \\
\hline \multicolumn{4}{|l|}{ Complicación readmisión } \\
\hline Absceso de pared & $1(2.0)$ & $0(0)$ & \\
\hline \multicolumn{4}{|l|}{ Tratamiento readmisión } \\
\hline Cirugía & $1(2.0)$ & $1(3.0)$ & \\
\hline Médico & $1(2.0)$ & $0(0.0)$ & \\
\hline
\end{tabular}

ción de herida quirúrgica (IHQ). ${ }^{4,14}$ Esto posiblemente explicado por la rapidez con que se ejecuta la intervención en nuestra institución, con la mayor parte de casos intervenidos antes de 24 horas (Cuadro 3).

El diagnóstico de la apendicitis complicada se basa en la sospecha clínica inicial con evaluación de laboratorio e imágenes, basándose en historia clínica, tiempo de evolución, cuadro clínico actual y progresión para realizar apendicectomía de urgencia laparoscópica 0 abierta, excepto en los casos en que hay dificultades para realizar diagnóstico diferencial. ${ }^{1,5,13-16} \mathrm{La}$ antibioticoterapia intravenosa estándar ha consistido en ampicilina más amikacina más clindamicina o metronidazol con efi- cacia y seguridad reconocida. En los estudios internacionales realizados, cada vez hay más evidencia sobre la eficacia y seguridad similares de la monoterapia, así como la transición a medicamentos por vía oral. ${ }^{17}$ En un estudio retrospectivo realizado en $2007,{ }^{5}$ en 8545 pacientes en 332 hospitales de Estados Unidos de América demostró menor estancia, así como menores costos de proceso de farmacia y costos totales hospitalarios comparado con triple terapia. Por otra parte, así como lo recomendado por la Asociación Americana de Cirugía Pediátrica y Peter \& Little, que en una reciente revisión sistemática sugiere monoterapia o doble terapia por su similar eficacia a triple terapia. ${ }^{6,7,10}$ En otro estudio que se comparó triple terapia versus monoterapia con Ertapenem, se obtuvieron mejores resultados en el grupo de monoterapia, al mismos tiempo que la colonización por bacterias resistentes también fue menor en este grupo. ${ }^{18}$

El diagnóstico de apendicitis aguda depende en gran parte de la exclusión de otras patologías similares clínicamente con ayuda laboratorial e imagenológica, que por separado no muestran utilidad importante. 1,2,4, El cuadro clínico es predictivo de apendicitis, como el caso del Score Pediátrico de Apendicitis, recomendándose en casos difíciles realización de ultrasonido y tomografía axial computarizada. ${ }^{2,3,20}$ Aunque la realización de estudios diagnósticos no es estándar en nuestra institución, el ultrasonido abdominal se realizó en menos de un sexto del total de pacientes, conteo leucocitario fue realizado en la mayoría de los pacientes. (Cuadro 2).

Entre las fortalezas de este estudio está en que es el primero realizado en Honduras comparando la eficacia de la monoterapia con la triple terapia antibiótica, lo que abre la posibilidad de otros estudios con mayor número de participantes para comprobar estos resultados y generar una recomendación que puede aumentar la calidad de atención por la menor necesidad de recursos logísticos y de suministros, sin detrimento de la eficacia terapéutica. Otra fortaleza de este estudio es que brinda información sobre aspectos clínicos y evolutivos generales del paciente pediátrico apendicectomizado. Entre las limitaciones, encontramos dificultades de suministro de Ertapenem, lo que obstaculizó la inclusión de mayor número de pacientes en este grupo, que habría generado conclusiones de mayor precisión respecto a potenciales efectos adversos. Una limitación importante es que los dos grupos fueron diferentes en cuanto a la evolución de la enferme- 
dad, con un mayor número de participantes con mayor evolución de los síntomas en el grupo monoterapia. Se recomienda la realización institucional de estudios con mayor número de pacientes para confirmar estos hallazgos. En conclusión, Ertapenem como monoterapia presentó una eficacia y seguridad similar al tratamiento estándar con tres antibióticos de apendicitis complicada, con ventajas logísticas y de cumplimiento sobre la triple terapia. Sin embargo, este estudio está limitado por que los dos grupos de estudio fueron diferentes en la evolución de la enfermedad.

\section{CONTRIBUCIONES}

Los tres autores concibieron y diseñaron el estudio. KS lideró la redacción del artículo. Todos los autores atendieron las recomendaciones editoriales. Todos los autores aprobaron la versión final del artículo.

\title{
REFERENCIAS
}

1. Henry M, Moss L. Appendicitis. En: Long S, Pickering L, Prober C, editors. Principles and practice of pediatirc infectious diseases. 3rd ed. Philadelphia, PA, USA: Churchill Livingstone; 2008. p. 626-9.

2. Lizardo J, Wildt D. Apendicitis Aguda ¿Ser o no ser? Revisión Bibliográfica Pediátrica. Rev Med Hondur. 2009;77(3):118-21.

3. Samuel M. Pediatric appendicitis score. J Pediatr Surg. 2009;37(6):87781.

4. Allister L, Bachur R, Glickman J, Horwitz B. Serum markers in acute appendicitis. J Surg Res. 2011;168(1):70-5.

5. Goldin AB, Sawin RS, Garrison MM, Zerr DM, Christakis DA. Aminoglycoside-based triple-antibiotic therapy versus monotherapy for children with ruptured appendicitis. Pediatrics. 2007;119(5):905-11.

6. Lee SL, Islam S, Cassidy LD, Abdullah F, Arca MJ. Antibiotics and appendicitis in the pediatric population: an American Pediatric Surgical Association Outcomes and Clinical Trials Committee systematic review. J Pediatr Surg. 2010;45(11):2181-5.

7. St Peter SD, Tsao K, Spilde TL, Holcomb GW, 3rd, Sharp SW, Murphy JP, et al. Single daily dosing ceftriaxone and metronidazole vs standard triple antibiotic regimen for perforated appendicitis in children: a prospective randomized trial. J Pediatr Surg. 2008;43(6):981-5.

8. Fraser JD, Aguayo P, Leys CM, Keckler SJ, Newland JG, Sharp SW, et al. A complete course of intravenous antibiotics vs a combination of intravenous and oral antibiotics for perforated appendicitis in children: a prospective, randomized trial. J Pediatr Surg. 2010;45(6):1198-202.

9. Puapong D, Lee SL, Haigh PI, Kaminski A, Liu IL, Applebaum H. Routine interval appendectomy in children is not indicated. J Pediatr Surg. 2007;42(9):1500-3

10. St Peter SD, Little DC, Calkins CM, Murphy JP, Andrews WS, Holcomb $\mathrm{GW}, 3 \mathrm{rd}$, et al. A simple and more cost-effective antibiotic regimen for perforated appendicitis. J Pediatr Surg. 2006;41(5):1020-4.

11. Aprahamian CJ, Barnhart DC, Bledsoe SE, Vaid Y, Harmon CM. Failure in the nonoperative management of pediatric ruptured appendicitis: predic- tors and consequences. J Pediatr Surg. 2007;42(6):934-8; discussion 938.

12. Glass CC, Rangel SJ. Overview and diagnosis of acute appendicitis in children.Semin Pediatr Surg. 2016;25(4):198-203. doi:10.1053/j.sempedsurg.2016.05.001

13. Taqi E, Al Hadher S, Ryckman J, Su W, Aspirot A, Puligandla P, et al. Outcome of laparoscopic appendectomy for perforated appendicitis in children. J Pediatr Surg 2008;43(5):893-5.

14. Wang X, Zhang W, Yang X, Shao J, Zhou X, Yuan J. Complicated appendicitis in children: is laparoscopic appendectomy appropriate? A comparative study with the open appendectomy--our experience. J Pediatr Surg. 2009;44(10):1924-7.

15. Whyte $\mathrm{C}$, Levin $\mathrm{T}$, Harris $\mathrm{BH}$. Early decisions in perforated appendicitis in children: lessons from a study of nonoperative management. J Pediatr Surg. 2008;43(8):1459-63.

16. Sakpal SV, Bindra SS, Chamberlain RS. Laparoscopic Appendectomy Conversion Rates Two Decades Later: An Analysis of Surgeon and Patient-Specific Factors Resulting in Open Conversion. J Surg Res .2012;176(1):42-9.

17. Loux TJ, Falk GA, Burnweit CA, Ramos C, Knight C, Malvezzi L. Early transition to oral antibiotics for treatment of perforated appendicitis in pediatric patients: Confirmation of the safety and efficacy of a growing national trend. J Pediatr Surg. 2016;51(6):903-907. doi:10.1016/j.jpedsurg.2016.02.057

18. Dalgic N, Karadag CA, Bayraktar B, Sancar M, Kara O, Pelit S, et al. Ertapenem versus standard triple antibiotic therapy for the treatment of perforated appendicitis in pediatric patients: a prospective randomized trial. Eur J Pediatr Surg. 2014;24(5):410-8. doi: 10.1055/s-0033-1352524

19. Hagendorf BA, Clarke JR, Burd RS. The optimal initial management of children with suspected appendicitis: a decision analysis. J Pediatr Surg. 2004;39(6):880-5.

20. Rentea RM, St Peter SD. Pediatric Appendicitis.Surg Clin North Am. 2017;97(1):93-112. doi:10.1016/j.suc.2016.08.009.

\begin{abstract}
Background: In Honduras there is no published evidence on the efficacy of different antibiotic regimens for post-surgical management of acute complicated appendicitis in children. Objective: To determine efficacy and safety of triple versus antibiotic monotherapy in pediatric patients with acute complicated appendicitis, Hospital de Especialidades, Instituto Hondureño de Seguridad Social (IHSS), Tegucigalpa, 2011-2013. Methods: Open clinical trial, two therapeutic schemes: Triple Therapy (Ampicillin+Amikacin+ Clindamicina, GroupTT) and Ertapenem (GroupE) for 7 days. Patients diagnosed with complicated acute appendicitis and subjected to open appendectomy were included. Patients were evaluated at the outpatient clinic, 7 days after surgery. Approval was obtained by the IHSS Research Ethics Committee. Chi-square test, Relative Risk, $95 \%$ confidence interval, and $p<0.05$ value were used to determine differences between groups. Results: 58 patients were included in GrupoTT and 29 in GrupoE, average age was 7.3 years (IC95\% 6.7-7.9) GroupTT and 8 years (IC95\% 7-9) GrupoE. The average evolution of the clinical picture was 31.6 hours GroupTT and 43.8 hours GroupE $(p=0.034)$. No significant differences were found regarding adverse effects of clinical significance, postoperative complications, intrahospital stay in days, or need for recourse/post-discharge complication readmission. Discussion: Monotherapy with ertapenem was similarly effective and safe as triple ampicillin+Amikacin+Clindamicin therapy currently used in the treatment of the paediatric patient with complicated appendicitis. The study is limited because the two study groups were different in the evolution of the disease.
\end{abstract}

Keywords: Anti-bacterial agents, Appendectomy, Appendicitis, Postoperative complications. 